\title{
Renforcer les compétences grâce à l'agriculture urbaine : rapport sur le projet Askîy
}

\author{
Wanda Martin, Ph. D., inf. aut.; Lindsey Vold, B. Sc. inf., inf. aut.
}

\author{
Cet article de recherche qualitative originale a fait l'objet d'une évaluation par les pairs.
}

Diffuser cet article sur Twitter

\section{Résumé}

Introduction : De nombreuses villes d'Amérique du Nord ont construit un environnement donnant accès à des aliments riches en calories et laissant peu de possibilités de mener une vie active. L'agriculture urbaine contribue à un environnement favorable grâce à la culture de végétaux destinés à l'alimentation, ce qui implique leur transformation, leur entreposage, leur distribution et leur compostage. Elle constitue un moyen d'augmenter la production alimentaire locale et d'améliorer ainsi la santé dans les collectivités. Cette étude avait comme objectif de comprendre comment la participation à l'agriculture urbaine peut contribuer à l'autonomisation de jeunes adultes et au renforcement des compétences à cultiver des aliments en ville.

Méthodologie : Il s'agissait d'une étude qualitative reposant sur sept participants (cinq Autochtones et deux non-Autochtones) de 19 à 29 ans, recrutés comme stagiaires dans un projet d'agriculture urbaine appelé " Askîy " à Saskatoon (Saskatchewan, Canada) en 2015. Nous avons utilisé un modèle d'étude de cas et une analyse qualitative pour décrire l'expérience des participants en fonction du Cadre des moyens de subsistance durables.

Résultats : L'approche collaborative a eu un effet majeur sur l'expérience des stagiaires, en particulier dans les liens qu'ils ont formés au fil de la planification, de la plantation, de l'entretien, de la récolte et de la vente des produits. Certains stagiaires ont changé leurs habitudes en matière d'épicerie et ont commencé à acheter plus de légumes et à demander où et comment sont produits les légumes. Tous les stagiaires étaient enthousiastes à l'idée de jardiner de nouveau l'année suivante, et certains d'entre eux planifiaient d'appliquer leurs connaissances et leurs compétences dans leur réserve d’origine.

Conclusion : Les programmes d'agriculture urbaine renforcent les compétences en permettant d'acquérir des habiletés qui vont au-delà de la culture d'aliments. Ils augmentent la production alimentaire locale et améliorent les compétences en littératie alimentaire, les relations sociales, l'activité physique et la fierté au sein de la collectivité.

Mots-clés : sécurité alimentaire, agriculture urbaine, autonomie, résilience, renforcement des capacités, capital social, cohésion sociale, cadre des moyens de subsistance durables

\section{Introduction}

L'agriculture urbaine est une intervention en santé communautaire qui permet d'augmenter la production alimentaire locale et d'améliorer les compétences en littératie alimentaire, les relations sociales, l'activité physique et la fierté au sein de la collectivité1. L'une de ces interventions, le projet Askîy, existe depuis 2014 grâce à CHEP
Good Food Inc. en partenariat avec le Saskatoon Food Council et l'Université de la Saskatchewan. Le projet Askîy a pu être financé par le programme Partenariats urbains de la Mosaic Company et d'Affaires autochtones et du Nord Canada. Les participants du projet étaient de jeunes adultes (« stagiaires ») qui ont cultivé des potagers urbains pendant l'été. Ils ont eux-mêmes choisi comme nom de projet Askîy, un mot

\section{Points saillants}

- Le projet Askîy a permis aux participants d'échanger des connaissances à propos de la culture des Premières Nations.

- Les stagiaires ont parlé d'emplois futurs, ont amélioré leurs compétences en communication et en relations interpersonnelles ainsi que leur confiance en leur propre corps, se sont fixé des buts, ont amélioré leur attitude envers la nourriture, ont changé leur alimentation, ont valorisé la nature et ont tissé des liens sociaux solides.

- L'un des défis a été de trouver un espace à cultiver doté d'un accès à l'eau et à l'électricité.

- Le programme renforce les compétences des jeunes adultes en leur offrant un endroit où appliquer les connaissances locales des Premières Nations, en créant un espace sécuritaire qui donne droit à l'erreur, en introduisant de nouvelles idées, en permettant l'autoapprentissage et en leur procurant des compétences utiles sur le marché du travail.

cri qui signifie " terre ». Ce projet favorise la croissance personnelle et incite les jeunes adultes autochtones comme non autochtones à contribuer à l'agriculture urbaine et à acquérir des compétences en culture d'aliments et en entrepreneuriat en vendant des produits au marché fermier de Saskatoon.

Cette étude, qui n'a reçu aucune subvention, a eu pour objet de comprendre l'effet de la participation des jeunes adultes à l'agriculture urbaine et de fournir des données pilotes en vue de plus amples études

Rattachement des auteures :

École de soins infirmiers, Université de la Saskatchewan, Saskatoon (Saskatchewan), Canada

Correspondance : Wanda Martin, École de soins infirmiers, Université de la Saskatchewan, 104 Clinic Place, Saskatoon (Saskatchewan) S7N 2Z4; tél. : 306-966-5429; téléc. : 306-966-6621; courriel : wanda.martin@usask.ca 
afin de faire progresser l'agriculture urbaine et de créer une économie alimentaire autonome. Les objectifs étaient d'explorer la manière dont le projet pouvait renforcer les compétences, les connaissances et les habiletés des jeunes adultes grâce à la culture d'aliments en ville ainsi que de leur apprendre ce que signifiait la production alimentaire urbaine. Nous espérons que les résultats de cette étude permettront à CHEP Good Food Inc. d'améliorer son travail en agriculture urbaine avec de jeunes adultes du centre-ville et d'améliorer les projets d'agriculture urbaine à Saskatoon.

\section{Définition du problème}

Avec cinq milliards d'habitants en milieu urbain prévus d'ici 2025 à l'échelle de la planète, les experts doutent de notre capacité à répondre à la demande en aliments nutritifs ${ }^{2}$. Actuellement, en Saskatchewan, $67 \%$ de la population vit en milieu urbain $^{3}$, et la population urbaine de la ville de Saskatoon devrait atteindre 500000 personnes d'ici $2023^{4}$. Il faut donc planifier afin de répondre à la demande croissante en aliments nutritifs. Environ $10 \%$ de la population (36 000 personnes) de la région sanitaire de Saskatoon se définit comme autochtone (Premières Nations et Métis) ${ }^{5}$ avec, au sein de ce groupe, $70 \%$ de personnes qui occupent un emploi, avec un revenu annuel moyen de $13000 \$$. Par ailleurs, dans les quartiers centraux de Saskatoon, comme celui de Riversdale, environ $57 \%$ des individus et des familles ont un revenu inférieur à $25000 \$$. Les nutritionnistes de la santé publique de la province estiment le coût annuel d'un panier d'épicerie nutritif (aliments sains conformes aux recommandations du document Bien manger avec le Guide alimentaire canadien) dans les grandes villes de la Saskatchewan à environ 10000 \$ par an, ce qui laisse aux personnes se trouvant dans les tranches inférieures de revenu très peu d'argent pour leurs autres frais de subsistance ${ }^{8}$. Étant données la croissance attendue de la population de Saskatoon et les difficultés rencontrées par les ménages à faible revenu, les professionnels de la santé publique peuvent contribuer davantage à la résilience en milieu urbain, surtout auprès des Premières Nations et des Métis, en fournissant des outils pour la production alimentaire urbaine. Cet objectif cadre avec le Plan stratégique 2013-2023 de la ville de Saskatoon, qui souligne les antécédents d'autonomie et de gestion de la ville et qui prend note que les résidents " produisent davantage d'aliments en ville $»^{4,}$ p. ${ }^{31}$. On parle là d'agriculture urbaine.

\section{Agriculture urbaine}

L'agriculture urbaine consiste aussi bien à entretenir des jardins dans différents endroits en ville qu'à faire du microélevage (poules, lapins et abeilles, etc.) ${ }^{9,10}$. Ces activités impliquent la participation des citoyens à la gouvernance du système alimentaire et offrent une possibilité de souveraineté alimentaire en milieu urbain. Ce mouvement de souveraineté alimentaire met en valeur le pouvoir politique et économique du système alimentaire et constitue une solution alternative au modèle néolibéral, qui privilégie les forces du marché plutôt que l'équité en santél1. Il renvoie à un processus d'expansion de la démocratie afin de régénérer des systèmes alimentaires locaux, autonomes, sains et écologiques respectant le droit à des conditions de travail et à un revenu décents ${ }^{12,13}$. Weiler et ses collaborateurs ${ }^{14}$ décrivent la façon dont la souveraineté alimentaire et l'équité en santé partagent une orientation commune qui vise à promouvoir l'épanouissement de l'humanité en uniformisant l'accès au pouvoir et à la circulation des biens grâce au système alimentaire. L'agriculture urbaine permet aux citoyens à la fois de participer directement au système alimentaire, d'acquérir un sentiment de pouvoir et de contrôle et d'améliorer leur santé.

\section{Perspective des systèmes}

La production alimentaire de qualité ne constitue que l'un des bienfaits de l'agriculture urbaine sur la santé, d'autres manières d'accéder à des moyens de subsistance durables susceptibles d'améliorer l'équité en santé étant un environnement bâti sain et un revenu suffisant ${ }^{1}$. Assurer une production alimentaire de qualité et un environnement bâti sain nécessite une perspective systémique. Le concept de moyens de subsistance durables a été utilisé traditionnellement en milieu rural et il renvoie à différents types de capitaux ou de ressources qui peuvent aider à se remettre d'un stress ou d'un choc $^{15}$. Afin de renforcer les moyens de subsistance durables, les professionnels de la santé publique peuvent envisager les différents secteurs qui utilisent les capitaux physiques, naturels, humains, sociaux et financiers décrits dans le Cadre des moyens de subsistance durables du ministère du Développement international du Royaume-Uni ${ }^{16}$. L'Organisation mondiale de la santé préconise des politiques et des programmes intégrés, basés sur une collaboration intersectorielle et capables d'assurer un approvisionnement alimentaire sain et durable, d'améliorer la cohésion sociale et d'apporter des bénéfices économiques et environnementaux ayant un effet positif sur l'équité en santé ${ }^{17}$. Les programmes qui vont intégrer ces secteurs et impliquer l'ensemble du système vont constituer des indicateurs importants de succès dans la contribution à l’amélioration de la santé urbaine.

Le Cadre des moyens de subsistance durables distingue cinq types de capitaux : capitaux physiques, naturels, humains, sociaux et financiers. Les capitaux physiques sont constitués des infrastructures de base en milieu urbain, dont l'approvisionnement en eau, les transports et l'accès à l'information ${ }^{16}$. En ville, ces capitaux peuvent aider à déterminer la manière dont les citoyens conçoivent le lieu où ils vivent et influencer les sentiments de fierté et de respect de soi, contribuant ainsi à une perception globale de bonne santé. Les capitaux humains sont constitués des connaissances, des compétences, de l'aptitude à travailler et d'une bonne santé susceptibles de contribuer à la recherche de ses moyens de subsistance $^{16}$. Les capitaux sociaux sont constitués des réseaux et de la capacité à se connecter de manière à favoriser la coopération ${ }^{18}$. Cela comprend plus particulièrement l'engagement communautaire, l'inclusion et la gestion du voisinage $^{16}$. Les capitaux financiers sont l'argent ou les équivalents disponibles pour adopter des stratégies de subsistance ${ }^{16}$. Ce sont en général les moins accessibles aux personnes qui ont le plus à gagner d'une amélioration de l'équité en santé ${ }^{19}$. Les capitaux naturels en agriculture urbaine sont constitués de ce qui augmente les possibilités d'exercice physique extérieur (comme les espaces verts) et offre des avantages notables pour la santé20. Il est essentiel d'améliorer les capitaux naturels en milieu urbain pour que la population soit en bonne santé, aujourd'hui comme demain.

\section{Le projet Askîy}

Les stagiaires ont travaillé dans les quartiers du centre-ville de Saskatoon, transformant une friche industrielle* grâce à plus de 300 contenants de plantation. Ils avaient

${ }^{*}$ Une friche industrielle est un ancien site industriel ou commercial susceptible d'être chimiquement, physiquement ou biologiquement contaminé24. 
postulé pour un emploi d'été de quatre mois dans un jardin urbain afin d'acquérir des compétences en production alimentaire, en leadership et en marketing sur le marché local. Des membres du College of Agriculture and Bioresources de l'Université de la Saskatchewan et de CHEP Good Food Inc. ont fourni régulièrement leur appui et ont enseigné de manière non formelle, par l'apprentissage pratique. Deux stagiaires avaient déjà certaines connaissances en jardinage et ont pris l'initiative d'encadrer leurs pairs. Les stagiaires ont utilisé un leadership collectif qui privilégiait la résolution de problèmes en groupe. Ils ont nommé l'espace kiscikânis (« jardin » en cri) et ont produit une grande variété de légumes, de fines herbes, de fleurs et de plants de tabac traditionnels. Le projet visait à éduquer les membres de la collectivité et à leur donner les connaissances et les habiletés nécessaires pour créer une économie alimentaire autonome grâce à l'agriculture urbaine ${ }^{21}$. La plupart des stagiaires n'avaient aucune expérience de jardinage, mais ils ont contribué avec succès à la production et à la vente des produits. Ils ont également partagé leurs connaissances avec d'autres personnes, dont de nouveaux Canadiens.

\section{Méthodologie}

Nous avons choisi un modèle d'étude de cas pour cette recherche et nous avons utilisé l'analyse thématique qualitative à multiples codeurs ${ }^{22}$. Le modèle d'étude de cas a été privilégié au moment de poser des questions sur le comment ou le pourquoi ${ }^{23}$. L'étude est fondée sur des entrevues ouvertes d'une heure avec les stagiaires du projet d'intervention (une entrevue pour chacun des sept). Alors qu'un groupe de discussion aurait plutôt permis de saisir la dynamique de groupe, les entrevues individuelles ont quant à elles permis aux participants de se questionner sur le sens de leur participation au programme de stage d'été et de l'exprimer. Nous avons reçu l'approbation sur le plan éthique du comité d'éthique de la recherche comportementale de l'Université de la Saskatchewan. Les coordonnateurs de programme ont informé les stagiaires de l'offre de participation et ont fixé la date, l'heure et le lieu des entrevues. Nous avons insisté sur le caractère volontaire des entrevues, et tous les stagiaires se sont engagés dans le processus. Nous avons utilisé des pseudonymes dans cet article pour personnaliser les citations des stagiaires.
Les entrevues individuelles ont été enregistrées puis transcrites pour analyse avec le logiciel d'analyse qualitative NVivo 11 (QSR International Pty Ltd., Melbourne, Australie). Dans le but d'explorer les effets de la participation au projet d'intervention, nous avons lu les transcriptions et catégorisé les données par thème, ce qui nous a fourni une description générale de l'expérience de participation au projet Askîy. Nous avons regroupé les concepts afin de faire émerger des thèmes et nous avons mis en regard ces thèmes émergents et le Cadre des moyens de subsistance durables ${ }^{16}$.

Cette confrontation des résultats au Cadre des moyens de subsistance durables a visé à mettre en lumière l'objectif initial du projet, qui était de créer une économie alimentaire autonome grâce à l'agriculture urbaine. L'optique de notre étude était en effet de parachever le projet Askîy en rendant compte de l'expérience des participants en agriculture urbaine et des limites à l'avancement de l'économie alimentaire autonome. Nos conclusions et les écarts que nous avons observés conduisent à élaborer d'autres échantillons et projets de recherche en lien avec la création d'une économie alimentaire autonome grâce à l'agriculture urbaine.

Huit stagiaires participaient à l'origine au projet Askîy, mais l'un d'eux a déménagé avant notre collecte de données. Les sept autres ont accepté de participer. Leur tranche d'âge était de 19 à 29 ans. Cinq étaient issus des Premières Nations ou d'origine métisse, et deux d'origine blanche. Cinq d'entre eux étudiaient à l'Université de la Saskatchewan ou venaient de terminer leur programme d'études (en enseignement, en arts et sciences, en génie chimique ou en gestion des ressources renouvelables). Deux participants étaient parents. Trois participants avaient une expérience de jardinage, mais aucun n'était confiant en ses compétences de jardinage au début du programme, tous se qualifiant de jardiniers débutants.

\section{Résultats}

\section{Le sens de l'agriculture urbaine}

Quoique cela ne fit pas précisément partie du Cadre des moyens de subsistance durables, nous voulions comprendre le sens que les stagiaires donnaient à l'agriculture urbaine et comment s'intégraient les connaissances des Premières Nations au projet
Askîy. Les participants ont déclaré que l'agriculture urbaine leur permettait de produire des aliments sains et de qualité tout en utilisant les espaces vacants. Pour les participants, l'agriculture urbaine signifiait la découverte, la flexibilité et la création d'un espace vert en milieu urbain. Pour Kara, l'une des participantes, l'agriculture urbaine allait au-delà d'un travail physique, contribuant à sa spiritualité :

C'est comme un arbre géant avec plein de racines et tout est lié. Je trouve que nous sommes tous tellement liés. Ce qui est génial avec l'agriculture urbaine, c'est qu'on peut trouver en quelque sorte le juste milieu. Dans notre société moderne, tu peux être tellement déconnecté de la terre et de la situation dans son ensemble et tu sais que la ville est bruyante et active mais que tu peux trouver un moment de paix dans le jardin. Je trouve cela très spirituel.

Pour Kara, la possibilité de s'engager à travailler la terre et à cultiver était plus importante que la production d'aliments. Les jardins ont permis de mettre à contribution et de concrétiser les connaissances locales ainsi qu'une reprise de contact avec la terre $^{25}$. La mémoire du jardinage et ses gestes quotidiens ont permis une plus grande connexion avec la culture et les connaissances traditionnelles. La culture de légumes a servi de catalyseur à la reprise de contact avec l'héritage des Premières Nations et avec la Terre grâce à la culture de plantes traditionnelles (p. ex. le tabac) et en étant près du sol, en le humant et en favorisant la croissance des plantes.

\section{Connaissances des Premières Nations}

Les stagiaires ont dirigé l'intégration des connaissances des Premières Nations au projet. L'équipe a intégré la langue crie au projet et des savoir-faire traditionnels en kiscikânis, dont la plantation en cercle avec quatre ouvertures, une dans chaque direction. Tammy, une participante, a expliqué que les ouvertures permettaient à " chaque grand-père et à chaque esprit d'entrer et de participer à la cérémonie ». L'équipe a invité les aînés de la communauté dans les jardins pour bénir la terre et certains membres ont sollicité les connaissances et les apprentissages des Premières Nations auprès des membres de leur famille et des aînés vivant dans leur réserve d'origine. Ils ont 
également appris l'importance d'avoir une énergie et des pensées positives lors de la plantation et de parler aux plantes, car chacune d'elle a un esprit. Grâce à cette manifestation d'amour, les stagiaires ont dit se sentir plus connectés, plus confiants et plus heureux.

De plus, ils ont planté quelques espèces indigènes et du tabac cérémoniel. Les Premières Nations utilisent le tabac pour les cérémonies et comme don, d'où son importance. Une stagiaire, Ana, a ainsi expliqué que le tabac est " aussi utilisé dans les cérémonies. De plus, si vous demandez des conseils à un aîné, si vous rendez visite à un chaman ou si vous allez dans une hutte à sudation, vous faites une offrande, généralement du tabac, à la personne responsable. " Le fait de participer au projet Askîy, qui incarne le point de vue des Premières Nations, a permis aux stagiaires d'échanger des connaissances, ce qui constitue un aspect essentiel du rajeunissement culturel. Donna a ainsi remarqué :

Je n'avais encore jamais vécu de bénédiction, alors c'était formidable d'en voir une. Nous sommes allés à un powwow. J'étais déjà allée dans un powwow, mais je n'avais jamais participé à un pow-wow de ce genre. C'est beaucoup de nouvelles choses pour moi. C'est vraiment intéressant. J'apprends beaucoup.

Les stagiaires ont affirmé que l'exposition aux savoirs traditionnels appliqués a constitué un apport important du projet, qui continuera à les accompagner. Pour certains, le projet Askîy s'est révélé la combinaison parfaite d'un moment, d'un lieu et d'une modalité pour qu'ils puissent voir le monde selon la perspective des Premières Nations.

\section{Culture des aliments - capitaux physiques}

Le principal défi du projet a été de trouver un lieu pour cultiver les aliments en toute sécurité. Certains terrains inoccupés le sont en raison de problèmes de contamination. L'équipe s'est finalement installée dans une zone de friche industrielle car aucun terrain alternatif n'était disponible pour la culture. Les plantes ont été placées dans 323 barils de plastique coupés sur le long et disposés sur des palettes de bois pour éviter le sol contaminé.

L'eau s'est également révélée un défi de taille. Les stagiaires ont sous-estimé la rétention de chaleur des barils de plastique, qui ont exigé plus d'eau que des jardins au sol, l'arrosage devant de plus se faire à la main. Le site n'ayant aucun accès à l'eau, l'équipe a acheté un gros réservoir d'eau que les membres du service des incendies de Saskatoon ont rempli bénévolement. Ce réservoir nécessitait une pompe à eau, et donc la fourniture d'électricité pour le jardin, ce qui a mené à l'achat d'une batterie. À chaque problème, l'équipe a travaillé à trouver une solution.

L'accès à l'information fait également partie des capitaux physiques. L'équipe a dit qu'un écart générationnel a entraîné une perte de connaissances, ce que décrit Ana :

Je n'ai pas grandi dans une famille qui m’a appris à jardiner ou qui avait des jardins. Ma grand-mère avait des platesbandes, mais elle n'a jamais cultivé d'aliments. C'est pourquoi je ne savais pas vraiment comment cultiver des aliments.

Il était rare de cultiver des aliments quand les stagiaires étaient jeunes, et ils ignoraient donc où aller afin de l'apprendre ou d'obtenir du mentorat. Ils ont en revanche trouvé des ressources sur Internet. Lorsqu'on a demandé à James ce qui serait utile afin de combler les lacunes en matière de connaissances, il a suggéré la création d'une trousse pédagogique ou d'une coopérative de travailleurs axée sur la culture des aliments, où les gens pourraient accéder facilement à des informations et à des ressources. Pour James, l'accès à l'information était aussi important que l'accès physique à la terre et à l'eau et a constitué un bon soutien pour essayer de nouvelles choses.

\section{Croissance personnelle - capitaux humains}

La possibilité de se découvrir soi-même peut être aussi considérée comme un capital important.

La plupart des stagiaires n'avaient aucune expérience de jardinage, ou alors le jardinage n'était pas pratiqué couramment dans leur famille. Les participants disent avoir augmenté leurs connaissances sur la culture des aliments et ont fait part de leurs inquiétudes sur l'origine de leur nourriture et sur la façon dont elle est cultivée. Ils ont aussi parlé de leurs changements d'attitude à l'égard de la nourriture. Ils ont commencé à valoriser les aliments locaux, peut-être parce qu'ils ont réalisé que la culture d'aliments demandait beaucoup de travail, ou encore en raison du goût des aliments cultivés localement et de la communauté croissante de personnes qui s'intéresse aux aliments locaux à Saskatoon.

Les participants ont signalé à quel point le projet les avait transformés. Ils ont parlé d'emplois futurs, de meilleures compétences en communication et dans les relations interpersonnelles, de confiance en leur corps, de la fixation d'objectifs, de leur attitude envers la nourriture, de changements dans leur alimentation et dans leurs valeurs. Kara a déclaré :

Quelques mois avant le stage, j'ai commencé à avoir une meilleure alimentation. J'ai aussi commencé à m'entraîner, j'étais donc un peu plus en forme au début du stage. Ensuite, tout cela a fait boule de neige quand j'ai eu le poste. Comme nous travaillons surtout à l'extérieur, c'est un emploi très exigeant et lorsque tu vois toute cette croissance, tu te dis, oh wow!

Le sentiment de fierté et d'accomplissement des stagiaires était très palpable lors des entrevues. On peut relier l'amélioration de la conception de la santé des participants à l'effet réparateur du jardinage urbain et des espaces verts urbains, grâce à des choix de vie plus équilibrés, à de l'activité physique et à une diminution remarquée du stress psychologique $^{26}$.

La croissance personnelle vécue par les participants s'est révélée émotionnellement fascinante. Doug a trouvé l'expérience particulièrement transformatrice :

J'ai commencé à jardiner à l'âge de 25 ans (24 plus exactement au début du stage), et c'est une leçon d'humilité d'apprendre qu'il ne me reste que quelques récoltes devant moi. Je considère sans doute ma vie en termes d'années, mais maintenant, je la pense davantage en termes de récoltes. Si je devais jardiner jusqu'à l'âge de 70 ans, il me resterait environ 45 ou 50 lots de ma salsa.

Comme plusieurs membres du groupe, Doug a changé sa façon de voir la vie, puisqu'il la voit maintenant en termes de récoltes et non d'années. En apprenant sur la culture des aliments, en travaillant sur la logistique 
quotidienne du jardinage dans une friche, en commercialisant les produits et en travaillant en équipe, les participants ont vécu une expérience enrichissante susceptible d'influencer, voire de façonner leur avenir.

\section{Effet sur les autres - capitaux sociaux}

Les participants ont également partagé l'expérience du jardin avec d'autres membres de la collectivité, en tant que groupe au service de nouveaux Canadiens. Cette association a été mutuellement bénéfique, car les participants ont reçu de l'aide pour la plantation et la récolte, tandis que les nouveaux Canadiens ont pu faire des rencontres et pratiquer l'anglais. De même, les participants autochtones et non autochtones ont vécu des interactions interculturelles. Les participants ont fait l'expérience de la réciprocité et de la confiance en dépit de leurs différences culturelles. Le jardinage communautaire a ainsi réuni des personnes d'horizons divers.

L'interaction sociale et l'échange d'idées ont été un moment fort pour ce groupe. Outre leurs compétences en jardinage et en marketing, les participants ont appris à travailler en équipe et à apprécier leurs forces - le plus important. L'un des stagiaires, Jeff, a comparé ses emplois précédents et cette expérience lorsqu'on lui a demandé ce qu'il retirait de ce projet :

Certainement la capacité de travailler en groupe. Dans la plupart de mes autres emplois, ce n'était pas autant fondé sur le travail en équipe. Nous étions un peu laissés à nous-mêmes ou quelqu'un nous disait quoi faire. Cet emploi était donc intéressant, car je n'avais jamais travaillé dans un groupe du genre. La voix de chaque personne était importante et la décision était universelle, au lieu qu'elle ne provienne que d'une personne.

Ce sentiment de cohésion est attribuable en partie à l'équipe de direction. Doug a souligné qu'il avait évolué en tant que leader, passant d'un rôle de " patron » à un rôle reposant davantage sur une attitude de respect :

[Dire aux gens quoi faire], ce n'est pas mon genre. Ça marchait peut-être pour une autre génération, mais je crois qu'avec les milléniaux, il faut faire preuve de respect pour se faire respecter, et on doit considérer les personnes comme des personnes.

Doug était conscient qu'il voulait le respect de l'équipe, ce qui impliquait être le premier sur les lieux, travailler fort, écouter les autres et respecter les idées et les contributions de chacun. Les deux chefs d'équipe avaient la même philosophie et ont favorisé un climat reposant sur une attitude d'équipe empathique.

Les capitaux sociaux du projet Askîy sont évidents : le travail d'équipe, l'écoute des habitants du quartier et l'ouverture à l'idée d'inclure d'autres groupes ont particulièrement enrichi cette expérience.

\section{La culture et la vente d'aliments - capitaux financiers}

Certains stagiaires ont souligné que la vente de légumes au marché de Saskatoon était rentable, mais il n'y a eu aucune discussion sur les coûts plus généraux du travail afin de produire les aliments, ou sur les coûts des intrants, comme le compost, l'eau et l'électricité. Il n'y a pas eu d'analyse coûts-avantages au moment des entrevues. Les stagiaires n’ont pas calculé les bénéfices en termes d'argent, mais plutôt en fonction des connaissances acquises, de ce qu'ils pourraient transmettre à d'autres collectivités et de la richesse de leurs relations avec les autres. Le projet n'étant pas autofinancé, sa réussite ne dépendait pas du profit réalisé grâce aux ventes. Il reste à savoir si l'agriculture urbaine est une entreprise viable pour de jeunes adultes sans expérience dans ce domaine. L'absence de discussions sur les finances peut s'expliquer par un manque de formation sur cet aspect de la production alimentaire dans le cadre du projet. Pour les stagiaires, la valeur du projet consistait à ce moment-là à accroître les connaissances à partager. Tammy a ainsi noté :

Je ne les vendrais pas à gros prix. J'ai envie de ramener ces connaissances chez moi afin d'aider ma communauté. Il y a des personnes qui n'ont pas beaucoup d'argent, pas assez pour s'acheter ces produits frais ou encore faire des dons. C'est pourquoi je ne les vendrais pas à gros prix.

Jeff a affirmé qu'une subvention était nécessaire pour les frais initiaux afin que l'entreprise soit viable, car il pense que les participants auraient besoin de plus de soutien au cours des trois premières années. Ana et Donna ont constaté que la culture des aliments était difficile, et même si de nombreux fermiers semblent heureux, la plupart sont découragés, endettés et vivent beaucoup de stress. Les stagiaires ont passé du temps avec un producteur du marché et ont été témoin des obstacles auxquels font face les producteurs à petite échelle, au-delà du travail physique sur le sol.

Des perspectives d'emplois futurs sont enviseagables pour les stagiaires à partir de leur expérience estivale hors du commun. Lorsqu'on a demandé à James si sa participation au projet l'aiderait à se trouver un emploi, il a répondu :

Probablement, oui. Je l'espère, car j'ai seulement une expérience de plongeur sur mon CV. En tant que stagiaire en agriculture, je pense que les gens voudront en savoir plus sur ce que j'ai fait ici.

Même si le projet n'offre aucun gain financier, les compétences acquises peuvent constituer un capital financier.

\section{Travailler en plein air - capitaux naturels}

Les participants ont parlé de capitaux naturels et ont exprimé combien le travail en plein air était plaisant. Ils ont parlé des insectes, se demandant si les organismes nuisibles mangeaient les plantes ou s'ils voyaient des insectes utiles. Ana a décrit l'augmentation d'insectes comme de la " biodiversité. Notre jardin attire beaucoup de papillons et d'abeilles, ce qui est vraiment génial ». Les participants ont mentionné d'autres capitaux naturels comme la réduction de l'effet d'îlot de chaleur, le recyclage de déchets alimentaires en compost et le potentiel de récupération d'eau de pluie afin de réduire l'écoulement des eaux pluviales.

Les jardins urbains servent d'espace collectif pour le transfert de connaissances et de traditions culturelles et pour la production de souvenirs socioécologiques sur la manière de cultiver des aliments et de naviguer avec succès entre les écueils de l'agriculture $^{27}$. En somme, la liste des services essentiels fournis dans les centres urbains par les jardins et les écosystèmes est longue en matière de santé et de bienêtre socioécologiques. 


\section{Analyse}

Cette recherche visait à comprendre comment le projet Askîy pouvait renforcer les compétences des jeunes adultes à cultiver des aliments en ville et à explorer chez les stagiaires le sens de l'agriculture urbaine. Ces derniers ont déclaré être fiers de ce qu'ils avaient accompli en participant au projet jusqu'à la fin. Ils ont passé du temps à faire pousser des plantes, à se sentir proches de la terre et à revenir à leurs racines tout en créant quelque chose d'écologique, de beau et d'utile. Ils ont fait quelque chose pour aider leur collectivité, ce qui a exigé une activité physique dans des conditions parfois difficiles, et ils se sont sentis à la hauteur. Ils ont prouvé que le travail acharné qu'est le jardinage permet d'acquérir des compétences de vie et que les récompenses valent tout ce travail ardu.

Leur apprentissage est allé au-delà du milieu urbain. Les participants venant des réserves ou ayant des liens avec celles-ci ont parlé de l'absence d'aliments frais en raison des coûts associés à l'envoi de fruits et de légumes dans les régions éloignées et, grâce à leur participation au projet, ils ont réalisé qu'il y avait un besoin dans leur communauté qu'ils voulaient combler. Une stagiaire a parlé du soutien des autres afin de créer un jardin communautaire chez elle. De plus, les participants ont ajouté qu'un jardin constituerait un atout précieux pour les collectivités isolées et pour les citadins qui n'ont aucun terrain, les familles à faible revenu ou les nouveaux immigrants. Ces résultats sont similaires à ceux de l'étude de Gray et ses collaborateurs $^{28}$ sur le rôle des jardins familiaux, selon laquelle les nouveaux jardiniers voulaient partager la beauté de produire et de consommer des légumes frais après avoir appris comment le faire par eux-mêmes.

Tous les participants au projet Askîy ont noté que le jardinage pouvait mener au changement social. Ana a dit que l'agriculture urbaine est un « mouvement social dans la bonne direction ». Certains participants ont parlé du jardinage comme d'un mode de résistance au système alimentaire industriel. Cela a permis un examen et une réflexion critiques sur le système alimentaire traditionnel. Cette conclusion n'est pas nouvelle, mais plutôt conforme à celle de Hansen ${ }^{29}$, qui parle de la façon dont les jardins communautaires peuvent constituer un site de résistance au système alimentaire mondial. Cette pratique de souveraineté alimentaire locale donne aux individus une sensation de puissance et de contrôle ainsi qu'une expérience de la propriété, dans un espace démocratique où ils partagent des buts sociaux, environnementaux et économiques $^{29}$. Les jardins peuvent constituer un point de convergence pour les membres de la collectivité désirant se réunir et définir ce qu'ils peuvent accomplir avec le pouvoir collectif ${ }^{30}$. Les forces de la mondialisation et une société de plus en plus individualiste exercent une pression sur nous, mais les collectivités et le jardinage communautaire peuvent être une source d'identité et de cohésion sociale. En tant qu'aire de loisirs, le jardin communautaire prolonge le progrès social, qui contribue à l'identité dans sa dimension de lieu de $v^{31}{ }^{31}$.

Même si nous n'avons pas utilisé une approche autochtone afin de mener notre étude, son application au programme reflète les valeurs des stagiaires et leur capacité à intégrer les savoirs des Premières Nations à leur stage d'été. Le projet Askîy a contribué à renforcer les compétences des jeunes, en leur permettant d'appliquer et de partager les connaissances des Premières Nations, en créant pour eux un espace sécuritaire qui donne droit à l'erreur, en les ouvrant à de nouvelles idées, en leur offrant des possibilités d'autoapprentissage et en leur apportant des compétences utiles sur le marché du travail. Cette approche au sein du projet est très importante pour la réussite et la viabilité globales de l'agriculture urbaine. Le programme de jardinage décrit par Gray et collaborateurs ${ }^{28}$ a ainsi prospéré lorsqu'ils ont utilisé une approche axée sur les capitaux : lorsque l'organisme responsable du programme s'est orienté vers le développement participatif, de plus en plus de jardiniers sont devenus propriétaires et se sont unis pour résoudre des problèmes locaux tout en développant le leadership local. Le projet Askîy est lui aussi un exemple d'approche de développement communautaire axée sur les capitaux, et CHEP Good Food Inc. n'évalue pas les résultats en kilogrammes de produits, mais plutôt en fonction du partage et des relations qui accompagneront les stagiaires tout au long de leur vie.

Les espaces verts et les jardins urbains constituent des capitaux sociaux et peuvent contribuer à un développement urbain durable en offrant une infrastructure saine et des aliments locaux aux citoyens, ce qui réduit à la fois le recours aux services produits à l'externe et l'empreinte écologique de la ville ${ }^{32}$. Nous concevons les jardins urbains comme faisant partie intégrante des infrastructures municipales. Ils constituent un capital collectif, en particulier pour les gens vivant en appartement ou n'ayant pas d'espace pour aménager un jardin, et ils procurent une meilleure qualité de vie. Le jardinage urbain favorise les grands espaces verts, encourage la biodiversité et soude les collectivités ${ }^{33}$.

Il existe des vulnérabilités dans le projet, avant tout économiques. Non seulement il n'était pas financièrement rentable, mais en matière d'apprentissage il n'a offert aucune compétence en gestion économique. À l'avenir, les concepteurs du programme pourraient fournir des informations aux stagiaires sur l'industrie de l'agriculture urbaine, comme le calcul des frais initiaux, la gestion des affaires et l'embauche du personnel. Les jardins urbains sont également à la merci des changements législatifs de zonage pour l'utilisation des terrains, des changements de propriété foncière pour l'élaboration d'autres ressources, des phénomènes météorologiques extrêmes et des changements politiques. La résilience sociale, humaine et physique acquise dans le cadre de l'agriculture urbaine présente un grand potentiel pour de futures recherches. Cette résilience a déjà été clairement démontrée, et ces vulnérabilités offrent des possibilités d'amélioration des programmes pour l'avenir.

\section{Points forts et limites}

Notre étude met en évidence les atouts d'une collectivité du centre-ville en mettant l'accent sur la mobilisation des jeunes et la possibilité de les laisser s'exprimer. Les futures recherches en évaluation peuvent s'inspirer de ces travaux afin de renforcer et d'élargir le projet Askîy. Notre étude comporte toutefois des limites. L'approche descriptive donne un aperçu du projet, alors qu'une étude phénoménologique pourrait fournir plus de précisions sur l'expérience vécue par les stagiaires au fil de leur travail et de leurs apprentissages collectifs. Le petit groupe était très positif et des informations essentielles ont émergé des entrevues. Une perspective critique pourrait déterminer les secteurs de croissance et les points à améliorer. Comme nous avons choisi le Cadre des moyens de subsistance durables après avoir réalisé les entrevues, les questions ouvertes ne nous ont pas permis d'obtenir autant de détails 
que si nous avions utilisé des questions plus précises pour respecter entièrement le cadre. Notre étude a néanmoins permis de saisir l'essence du projet et contribué à démontrer aux stagiaires la valeur du développement communautaire fondé sur les capitaux.

\section{Conclusion}

La principale force du projet réside dans ses capitaux humains et sociaux, mais tous les capitaux liés aux moyens de subsistance durables étaient présents. Les personnes qui ont participé au projet en 2015 ont fait preuve d'un grand potentiel en ce qui a trait au partage avec leurs réseaux des connaissances acquises auprès de CHEP Good Food Inc. Le renforcement de la résilience locale et de l'autonomisation contribuent à la vision d'un avenir meilleur. La création de systèmes alimentaires communautaires permet non seulement d'aider la population à produire des aliments, mais aussi de la reconnecter avec la nature et sa culture. La prochaine étape de la recherche va consister à établir des indicateurs de résultat afin de mesurer les effets de programmes de jardinage similaires, indicateurs reflétant non seulement les résultats directs du jardinage, mais aussi le capital social, le transfert et l'échange de connaissances et l'autonomisation personnelle.

\section{Remerciements}

Nous aimerions remercier les membres du personnel de CHEP Good Food Inc., ainsi que Grant Wood du College of Agriculture and Bioresources de l'Université de la Saskatchewan, d'avoir facilité ce projet.

\section{Conflits d'intérêts}

Les auteurs déclarent n'avoir aucun conflit d'intérêts.

\section{Contributions des auteures et avis}

WM a conçu l'étude et interprété les données. Les deux auteures ont contribué à la rédaction du manuscrit et à sa révision critique et ont lu et approuvé la version finale.

Le contenu de cet article et les opinions qui y sont exprimées n'engagent que les auteures et ne sont pas forcément représentatifs de la position du Gouvernement du Canada.

\section{Références}

1. Lovell R, Husk K, Bethel A, Garside R. What are the health and well-being impacts of community gardening for adults and children: a mixed method systematic review protocol. Environ Evid. 2014;3(1):20. En ligne à : https://environmentalevidencejournal .biomedcentral.com/articles/10.1186 /2047-2382-3-20

2. MacRae R, Gallant E, Patel S, Michalak M, Bunch M, Schaffner S. Could Toronto provide $10 \%$ of its fresh vegetable requirements from within its own boundaries? Matching consumption requirements with growing spaces. J Agric Food Syst Community Dev. 2010;1(2):105-127.

3. Statistique Canada. Mégatendances canadiennes - Un Canada de plus en plus urbain [Internet]. Ottawa (Ont.) : Statistique Canada; 2015 [consulté le 11 novembre 2015]. En ligne à : http:// www.statcan.gc.ca/pub/11-630-x/11 -630-x2015004-fra.htm

4. City of Saskatoon. Strategic plan 2013-2023. Saskatoon (SK) : City of Saskatoon; 2013.

5. Community view collaboration. First Nations and Métis Population. Community view collaboration; 2014. En ligne à : http://www.communityview .ca/pdfs/2014_SHR_FirstNations_ Metis.pdf

6. Gendron F, Hancherow A, Norton A. Exploring and revitalizing Indigenous food networks in Saskatchewan, Canada, as a way to improve food security. Health Promot Int. 2017; 32(5):1-10. doi : 10.1093/heapro/daw013.

7. City of Saskatoon. Neighbourhood profiles: Riversdale [Internet]. Saskatoon (SK): City of Saskatoon; 2015. En ligne à : https://www.saskatoon.ca/sites /default/files/documents/community -services/planning-development /research/neighbourhood-profiles /riversdale.pdf

8. Saskatchewan Food Costing Task (SFCT) Group. The cost of healthy eating in Saskatchewan 2012. Saskatoon (SK) : SFCT Group; 2012. 24 p.
9. La Rosa D, Barbarossa L, Privitera R, Martinico F. Agriculture and the city: a method for sustainable planning of new forms of agriculture in urban contexts. Land Use Policy. 2014;41: 290-303.

10. Smit, J. and J. Nasr, Urban agriculture for sustainable cities: using wastes and idle land and water bodies as resources. Env Urbanization. 1992; 4(2):141-152.

11. Wittman H, Desmarais AA, Wiebe N (dir.). Food sovereignty in Canada. Halifax (NS) : Fernwood Publishing; 2011. 232 p.

12. Blouin, C, Lemay J-F, Ashraf K, Imai J, Konforti L. Local food systems and public policy: a review of the literature [Internet]. Montréal (QC) : Équiterre and The Centre for Trade Policy and Law; 2009. En ligne à : https:// foodsecurecanada.org/sites/foodsecure canada.org/files/localfoodsystemsand publicpolicy_sept2009_0.pdf

13. Pimbert M. Towards food sovereignty: reclaiming autonomous food systems. [Internet]. London (UK) : The International Institute for Environment and Development; 2009. [consulté le 14 septembre 2017] En ligne à : https://www .iied.org/towards-food-sovereignty -reclaiming-autonomous-food-systems

14. Weiler AM, Hergesheimer C, Brisbois B, Wittman H, Yassi A, Spiegel JM. Food sovereignty, food security and health equity: a meta-narrative mapping exercise. Health Policy Plan. 2015;30(8):1078-1092.

15. Scoones, I., Livelihoods perspectives and rural development. J Peasant Stud. 2009;36(1):171-196.

16. Department for International Development (DFID). Sustainable livelihoods guidance sheets. London (UK) : DFID; 1999. $150 \mathrm{p}$.

17. de Leeuw E, Tsouros AG, Dyakova M, Green G. Healthy cities: promoting health and equity-evidence for local policy and practice. Copenhagen (DK) : Organisation mondiale de la santé; 2014. 32 p. 
18. Morse S, McNamara N. The theory behind the sustainable livelihood approach. In: Morse S, McNamara N, editors. Sustainable livelihood approach: a critique of theory and practice. London (UK): Springer Science and Business Media; 2013:15-60.

19. Marmot M, Friel S, Bell R, Houweling TA, Taylor S, Commission on Social Determinants of Health. Closing the gap in a generation: health equity through action on the social determinants of health. Lancet. 2008; 372(9650):1661-1669.

20. Leake, J.R., A. Adam-Bradford, and J.E. Rigby, Health benefits of 'grow your own' food in urban areas: implications for contaminated land risk assessment and risk management? Env Health. 2009;8(Suppl 1)S6. doi : 10.1186/1476-069X-8-S1-S6.

21. Severight-Dumais K. Urban agriculture: the askîy project [Internet]. 2015 [consulté le 20 avril 2016]. En ligne à : http://www.chep.org/en/programs /urbanag

22. Patton MQ. Qualitative research and evaluation methods (2e éd.). Thousand Oaks (CA): Sage Publications; 1990.

23. Yin RK. Case study research: design and methods. Beverly Hills (CA) : Sage; 1984.

24. Greenberg, M., Should Housing Be Built on Former Brownfield Sites? American Journal of Public Health. 2002;92(5):703-705.

25. Beilin R, Wilkinson C. Introduction: governing for urban resilience. Urban Studies. 2015;52(7):1205-1217.

26. Kabisch NS, Qureshi S, Haase D. Human-environment interactions in urban green spaces-a systematic review of contemporary issues and prospects for future research. Env Impact Assess Rev. 2015;50(Jan):25-34.

27. Barthel S, Parker J, Ernstson H. Food and green space in cities: a resilience lens on gardens and urban environmental movements. Urban Studies. 2013;52(7):1321-1338.
28. Gray L, Guzman P, Glowa KM, Drevno AG. Can home gardens scale up into movements for social change? The role of home gardens in providing food security and community change in San Jose, California. Local Env. 2014;19(2): 187-203.

29. Hansen Y. Growing community: community gardens as a local practice of food sovereignty. Dans : Wittman $\mathrm{H}$, Desmarais AA, Wiebe N (dir.), Food sovereignty in Canada. Halifax (NS) : Fernwood; 2011. p. 151-168.

30. Gottlieb R, Joshi A. Food justice. Cambridge (MA) : MIT Press; 2010. $304 \mathrm{p}$.

31. Forrest R, Kearns A. Social cohesion, social capital and the neighbourhood. Urban Studies. 2001;38(12):2125-2143.

32. McPhearson T, Andersson E, Elmqvist T, Frantzeskaki N. Resilience of and through urban ecosystem services. Ecosystem Services. 2015;12(Avr): 152-156.

33. Mougeot LJ (dir.). Agropolis: The social, political and environmental dimensions of urban agriculture. London (UK) : Earthscan, International Development Research Centre; 2010. $308 \mathrm{p}$. 